\title{
Triggered star formation in spiral arms
}

\author{
Eric E. Martínez-García ${ }^{1}$, Rosa Amelia González-Lópezlira ${ }^{1}$ \\ and Gustavo Bruzual-A. ${ }^{2}$ \\ ${ }^{1}$ Centro de Radioastronomía y Astrofísica, UNAM, México \\ ${ }^{2}$ Centro de Investigaciones de Astronomía, Venezuela
}

\begin{abstract}
We present preliminary results for six spiral galaxies from a sample of 25, where we have used the method developed by González \& Graham (1996) to search for and analyze azimuthal color gradients across spiral arms. The six galaxies analyzed here are NGC 1703 (SBrb), NGC 3001 (SABrsbc), NGC 3059 (SBrsbc), NGC 3513 (SBrsc), NGC 4593 (RSBrsb), and NGC 4603 (SAsc).

NGC 1703 : We found one azimuthal color gradient in NGC 1703. Star formation was traced with the reddening free parameter $\mathrm{Q}$, and the dust lane was located with the $(\mathrm{g}-\mathrm{J})$ color. This gradient lies at a distance of $1.45 \mathrm{kpc}$ from the center of the galaxy. In order to get some physical parameters of the star formation processes that are taking place in the spiral arms of the galaxies in our sample, we compared the observed Q profiles with the stellar population synthesis models of Bruzual \& Charlot (2003). The fitted Q model is shown in figure 1. If one assumes that stars form in the site of the shock, and that they age as they move away from this birthsite, then distance from the dust lane (at constant radius) parameterizes stellar age. In fact, stretching the model $\mathrm{Q}$ to the fit the data fixes the ratio between the distance and the age of the stellar population. If, in addition, the rotational velocity is known, it is possible to find the angular velocity of the spiral pattern. The spiral pattern speeds derived from the gradients (under the assumption that star formation is triggered by the density wave) yield theoretical resonance positions that are coincident with the observed spiral end points, in 3 out of 6 spirals. It would be hard to avoid the conclusion that disk dynamics and star formation are fundamentally related in these objects.
\end{abstract}

Keywords. star formation, spiral arms, density wave triggering

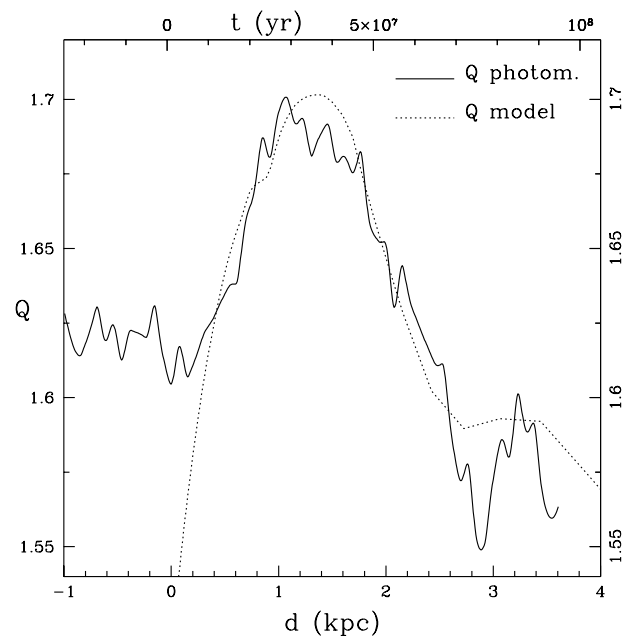

Figure 1. The fitted Q model (dotted line) compared to the observations (solid line). The zero distance indicates the location of the dust lane. 